Chapter 28

\title{
ESTUARINE CURRENTS AND TIDAL STREAMS
}

\author{
Roderick Aignew \\ Lecturer in Hydraulics, Civil Engineering Department \\ Imperial College of Science and Technology, London
}

\section{INTRODUCPION}

Fresh water spreading out from the mouth of a river as it onters a salt sea may preserve its identity for a considerable distance on the surface if wind-generated waves, longshore currents and tidal streams are capable of producing only weak mixing. Fig. I shows the threedimensional shape of a fresh-water tongue overlying more dense salt water, derived by Takano (1954) on the assumption of constant eddy viscosity and constant density in the fresh water layer, below which the density increases according to an assumed law, making an asymptotic approach to the density of salt water. Takano's model is thus a water jet ontraining salt from around and below it.

Salt or brackish water may penetrate along the deep channels of an estuary in the shape of a wedge complementary to the fresh water tongue, the salt wedge retreating seawards as heavy rainfall increases the river discharge, and advancing in dry weather intervals. Tidal streams cause a regular oscillation of both fresh and brack water in flood and $\mathrm{ebb}$ directions but the seasonal movements of the sloping boundary between fresh and salt water may still be important in low-lying delta regions. Strong tidal streams lead to intense mixing, when neither a fresh water tongue nor a salt wedge can be distinguished, but the isohalines (salinity contours) preserve the general wedge pattern - see Figs. 3 to 6.

In the upper reaches of an estuary it is possible to study the effect of the tidal motion by treating it as a simple harmonic perturbation of the uni-direotional river flow. Even in the middle portion of the estuary where there is reversal of the horizontal motion, one may seek a "quasi steady" solution for the net effect (seaward movement of fresh water) while allowing for the increased turbulence due to tho tidal action. At the seaward end of the estuary there is little deviation from the astronomical tidal rhythm, so the problem reduces to simple harmonic osoillations of salt water. Higher harmonics may be introduced as an extension of the simple solution. For a first approximation it is auficient to consider flow in the longitudinal vertical plane, to assume that the pressure distribution is hydrostatic as in long wave theory, and even to neglect inertia terms when investigating net effects. 

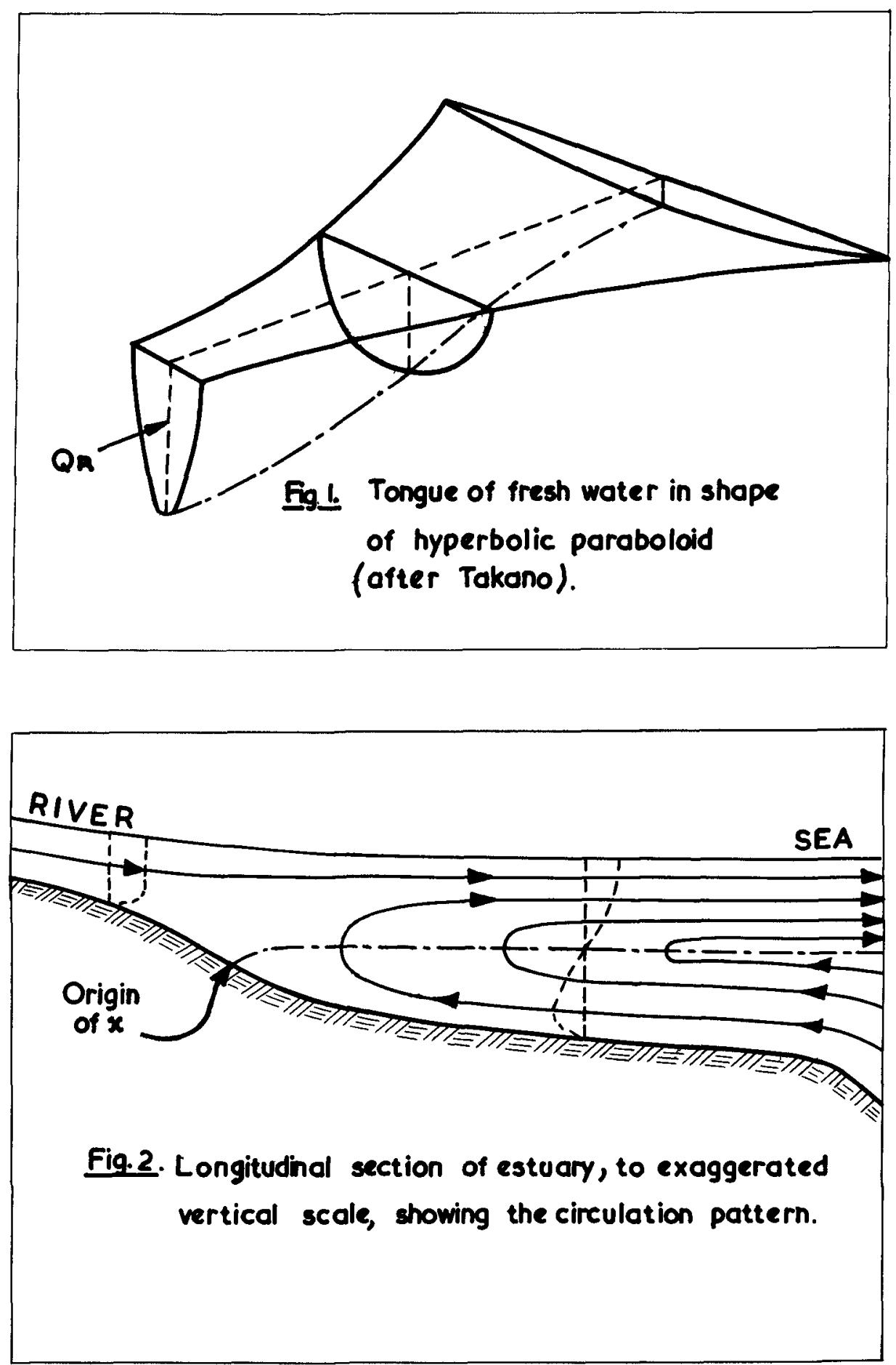
COASTAL ENGINEERING
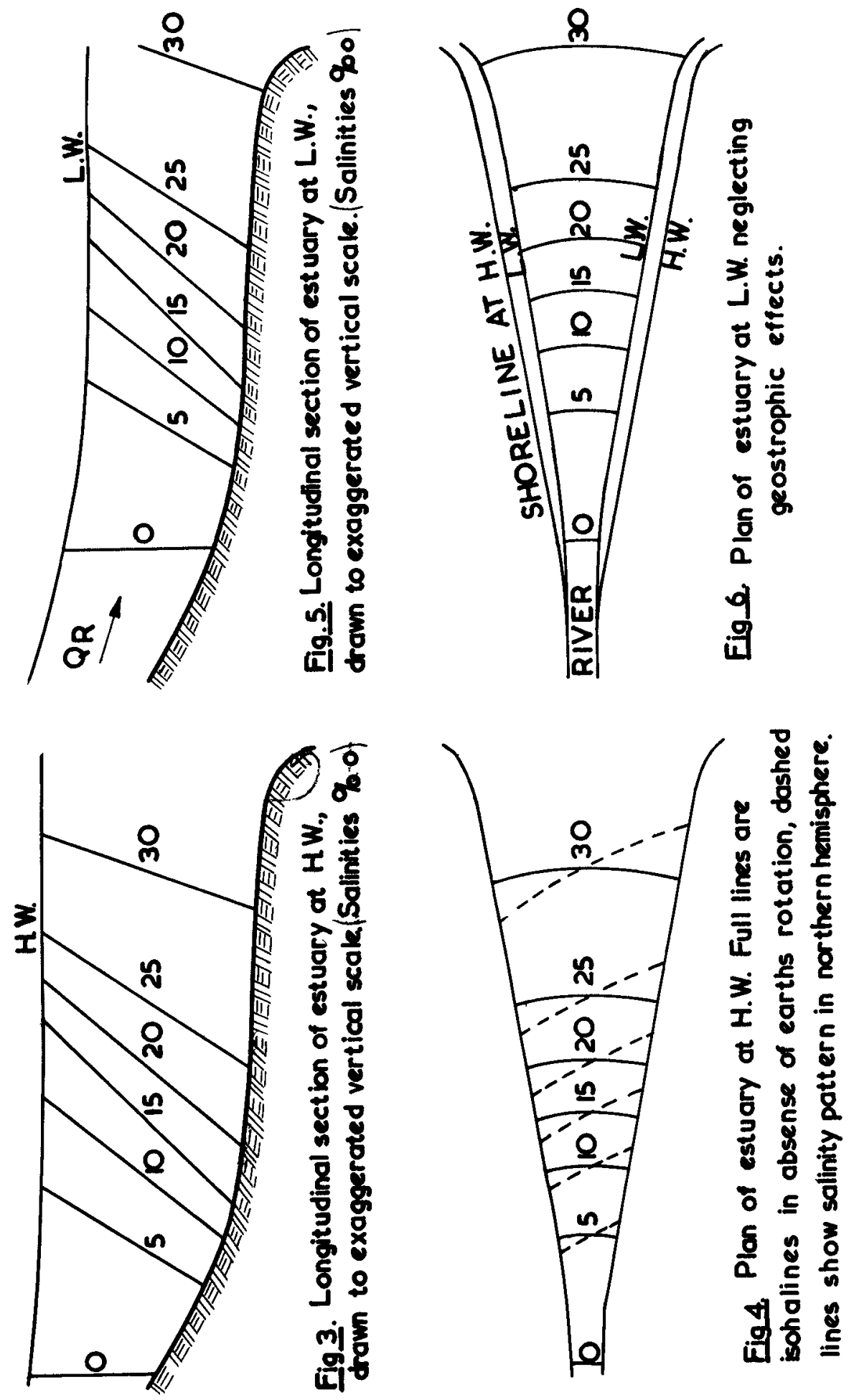


\section{ESTUARINE CURRENTS AND TIDAL STREAMS}

\section{DENSITY DISTRIBUTION}

For engineering purposes we may regard the oea as an infinite reservolr of galt water, its edges being diluted by fresh river water and rain while the balance is maintained by evaporation from its surface to the atmosphere. We postulate a slow current of seawater landwards along the bed, its density being decreased by vertical diffusion and mixing, and a surface flow seawards of fresh water being gradually rendered brackish by salt rising from below. This circulation is illustrated in Fig. 2 by full-drawn streamlines, the broken lines being profiles of longitudinal velocity. The hain dotted line indicates the surface of zero net motion in the longitudinal direction, and is obviously a place where high shear stresses may be expeeted, even exceeding the bed shear stress. This is a valid picture even when tidal motion is superimposed, although then the surface of zero net motion must be defined by averaging over a tidal period; it has a real existence only near the instants of "slack water". O'Brien (1952) suggested that landward velocity near bed is approximately C. $/ \mathrm{H} . \mathrm{D}$, where $C=$ Chezy coefficient and $D=\frac{H}{2 p} \cdot \frac{\partial p}{\partial x}$

In many estuaries it is observed that the arerage salinity over a cross-gection, and hence the average density if temperature differences can be neglected, increases from river to sea in nearly linear fashion in the middle reaches (Fig. 7 ), the rate of increase being smaller near the river $(x=0)$ and the sea $(x=I)$. This linear increase is related to the fact that the maximum velocity of the tidal atream $\bar{U}$ scarcely changes along the estuary* so the intengity of mixing shows little variation. The water and dissolved salt clearly oscillates a distance $X_{0}=\frac{y_{f}}{6}$ which 1 is usually a smali fraction of the length $I_{0}^{0}$ Wor exanple, if $\overline{\mathrm{U}}=1.4 \mathrm{~m} / \mathrm{sec}$. or approximately $3 \mathrm{kmots}$, and the angul\&r velocity of the lunar semi-diurnal tidal stream Is $\omega=0.00014 \mathrm{rad} / \mathrm{sec}$., then $X_{0}=10 \mathrm{~km}$, whereas $I \approx 80 \mathrm{~km}$ for a typical estuary.

Fig. 7 shows the average density distributions at slack water after high water (change from flood to ebb), and slack water after low water (ohange from ebb to flood), on the assumption that

$$
\bar{p}=p_{0}+\Delta p \cdot \sin ^{2} \frac{\pi \cdot x}{2 \cdot L}
$$

* Indeed, Pillabury (1939) inferred from the Delaware, and Otter and Day (1960) showed more rigorously with application to the Thames, that 0 is constant in a long estuary, whose breadth increases exponentially with $\mathbf{x}$. 
where $p_{0}=$ density of fresh water entering from river and $\Delta p=$ density difference between sea and fresh water. This density distribution fits the Thames data quoted by Inglis and Allen (1957), but other analytical curres such as the Gaussian integral and the hyperbolic tangent would also be suitable.

There is also an inorease of density from surface to bed, whioh is nearly linear in a well-mixed estuary, as indicated by the isohalines in Figs. 3 and 5 . Unless there is a deep channel through tidal flats there is intle variation of density in the transverse direction. The effect of the Earth's rotation is to tilt the isohalines (Fig. 4) much more than the water surfaoe. Assuming longitudinal translation of the isohalines without rotation, we now seek for a suitable law describing variation of the vertical density difference along the estuary.

Defining the salt concentration $e=\frac{\text { Mass of salt }}{\text { Mass of water }}$ at a point, the density is $p=(1+c) \cdot p_{0}$, and the vertioal flux or transfer of salt per unit area is c.p.W $=-p N_{\text {Salt }} \cdot \frac{\partial c}{\partial z}$ where $\mathrm{N}_{\text {Salt }}$ is the vertical exchange coefficient for salt, or the eddy diffusion ooeffioient. The Reynolds shear stress, or vertical flux of momentum, is similarly $\tau=-\overline{p \cdot w}=p \cdot N_{\text {Momentum }} \cdot \frac{\partial U}{\partial z}$, and $\mathbb{N}_{\text {Momentum }}$ is often called the eddy viscosity. ooefficient. Here $U$ and $W$ are the time-mean relocities, $u$ and $w$ the velocity fluctuations, in the longltudinal and rertical directions respectively. Jacobsen's method of averaging over a tidal cycle then yields the equations

$$
\begin{gathered}
U \cdot \frac{\partial c}{\partial x}=\frac{\partial}{\partial z}\left(N_{\text {Salt }} \cdot \frac{\partial c}{\partial z}\right) \\
\text { and }-g \frac{\partial p}{\partial x}=\frac{\partial^{2} \tau}{\partial z^{2}}=\frac{\partial^{2}}{\partial z^{2}}\left(p \cdot N_{\text {Momentum }} \cdot \frac{\partial U}{\partial z}\right)
\end{gathered}
$$

Extensive measurements have shown that the eddy coefficients are unequal, their ratio

$$
\frac{\text { Nomentum }_{\text {Mom }}}{\text { NSIt }_{\text {Sal }}}=\gamma
$$

being a funotion of the local Richardson number $R_{i}=\frac{-g \cdot \frac{\partial p}{\partial !}}{\mathrm{P} \cdot\left(\frac{\partial I}{\partial z}\right)^{2}}$ by fluid turbulence is wholly devoted to mixing (i.e. increasing potential energy of variable density fluid), and $\gamma>\operatorname{Ri}$ if some energy is dissipated by fluid visoosity. 

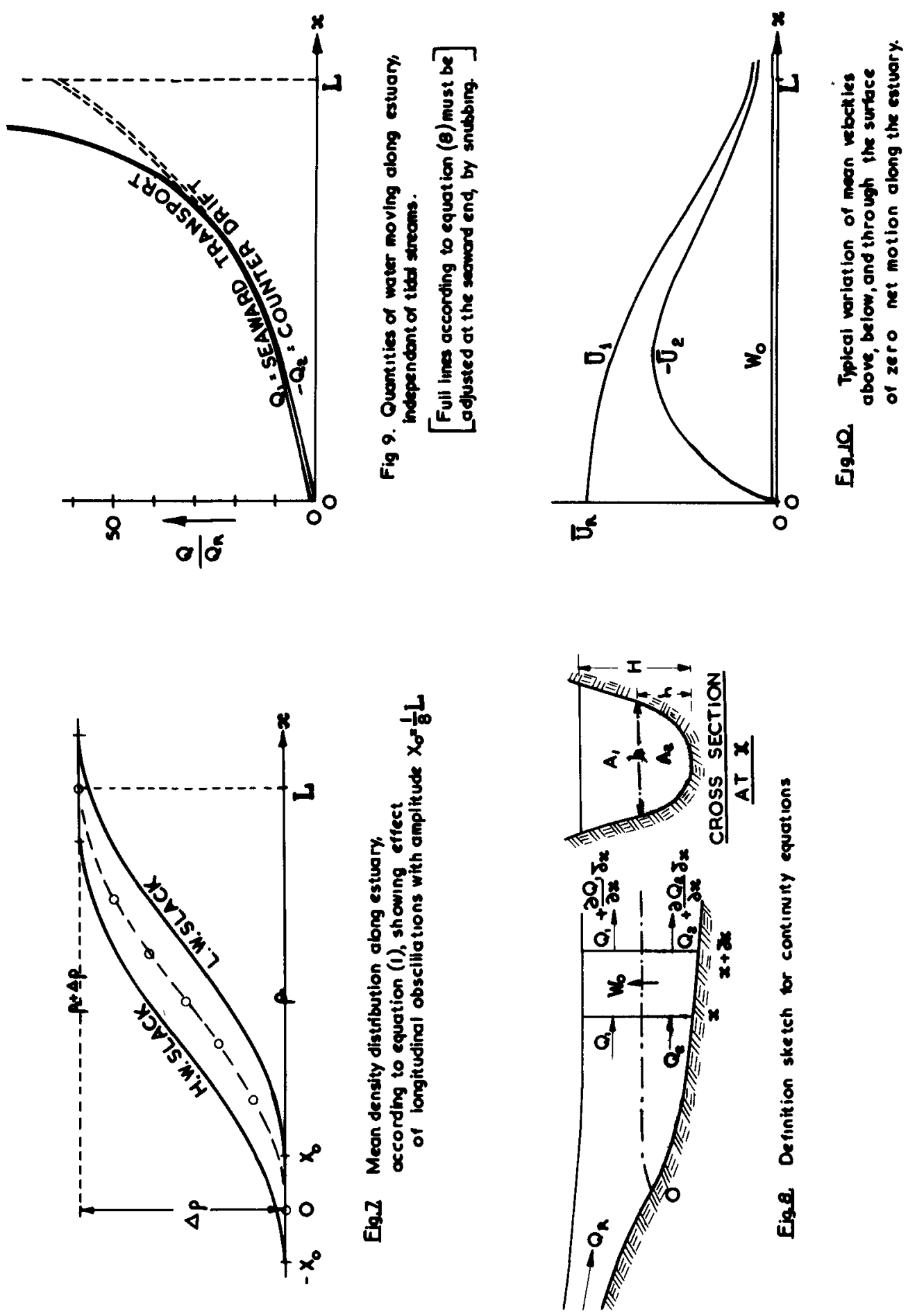
With specified velocity and density distributions, Taylor showed that stable internal waves are possible when $\mathrm{RI}>0.25$, but no waves can exist if $\mathrm{RI}<0.25$. Other workers have derived different stability criteria.

Let us assume that the vertical distributions of $\mathbb{N}_{\text {Momentum and }} \mathrm{N}_{\text {Salt }}$ are similar, hence the ratio $\gamma$ does not vary with z. Take $\mathrm{N}_{\text {Momentum }}=K \cdot U_{*} \cdot 2 \cdot\left(1-\frac{z}{H}\right)$

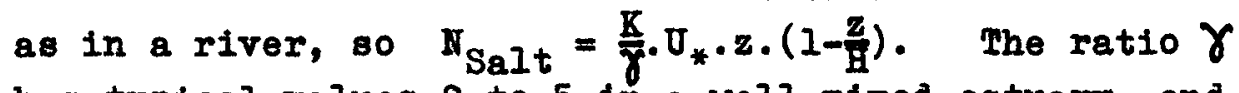
has typical values 2 to 5 in a well-mixed estuary, and 20 to 50 in a atably stratified estuary with a salt wedge.

Now $\frac{\partial c}{\partial z}=\frac{\partial p}{\partial z} \approx-\frac{\delta p}{H}$ if the density difference between bed $(z=0)$ and $\operatorname{surface}(z=B)$ is $\delta p$, a function of $x$. Also $\frac{\partial c}{\partial x}=\frac{\partial p}{\partial x} \approx \frac{\partial \bar{p}}{\partial x}=\frac{\pi . \Delta p}{2 . L}$. sin $\frac{\pi x}{L}$ from (I). The advection term $U \cdot \frac{c c}{x}$ in (2) obviously changes sign in the vertical. To get an idea of its effect, assume zero net velocity at mid-depth and surface velocity $\mathrm{V}_{\mathrm{H}}$ equal and opposite to velocity near the bed, thus $U=\mathrm{U}_{\mathrm{H}}\left(2 \frac{\mathrm{Z}}{\mathrm{H}}-\mathrm{I}\right)$. Substitution in equation (2) gives:-

$$
\begin{aligned}
U_{H} \cdot\left(2 \frac{z}{H}-1\right) \cdot \frac{\pi \cdot A p}{2 \cdot L} \cdot \sin \frac{\pi x}{L} & =\frac{\partial}{\partial z}\left[\frac{k \cdot U_{n}}{\gamma} \cdot\left(z-\frac{z^{2}}{H}\right) \cdot\left(-\frac{\delta p}{H}\right)\right] \\
& =\frac{k \cdot U_{x}}{\gamma \cdot H} \cdot\left(2 \cdot \frac{z}{H}-1\right) \cdot \delta
\end{aligned}
$$

whence

$$
\delta_{p}=\frac{\pi \cdot Y \cdot H}{2 \cdot K \cdot L} \cdot \frac{U_{H}}{U_{*}} \cdot \Delta p \cdot \sin \frac{\pi x}{L}
$$

Hence the vertical density difference is greatest in the middle reaches. It is zero at the river but not quite zero at the seaward end of the estuary, so that (4) is not reliable when $x \rightarrow I$.

If $P_{1}=$ mean denaity of water above surface of zero net motibh and $p_{2}=$ mean density of water below surface of zero net motibh, at section $x$, then the density difference between the two layers is $\rho_{2}-P_{2} \approx y_{2} .5$.

Typical values of the longitudinal and vertical den$\frac{\Delta p}{p_{0}} \approx \frac{30}{1000}$ and $\frac{\delta p_{M x}}{p_{0}} \approx \frac{2}{1000}$ in a well-mixed estuary.

\section{MEAN VELOCITIES OF CIRCULATION}

The equation of continuity for quasi-steady conditions states that the volume of water between two crosssections is constant, neglecting precipitation, evaporation, and tidal motion. Referring to Fig. 8, let 


\section{ESTUARINE CURRENTS AND TIDAL STREAMS}

$Q_{R} \quad=$ discharge of fresh water from river

$Q_{1}=A_{1} \cdot \bar{U}_{1}=$ flow above surface of zero net motion at $Q_{2}=A_{2} \cdot \bar{v}_{2}=$ " below " " " section

Discharge and velocity are measured positive in the direction of $x$ increasing, 1.e. from river to sea. Let $h=$ height above bed and $b=$ bread th of surface of zero net motion, through which there is an upward velocity $W_{0}$ meaned across the breadth, at seotion $x$.

Between 0 and $x$ the continuity requirement gives

$$
Q_{1}+Q_{2}=Q_{R}
$$

Considering the space between sections $x$ and $(x+\delta x)$ above the surface of zero net motion in Fig. 8, the water. entering per unit time is $Q_{1}+W_{0} \cdot b . \delta x$, and the water

leaving per unit time is $Q_{1}+\frac{\partial Q_{1}}{\partial x} \cdot \delta x$, whence

$$
\left.\begin{array}{rl}
\frac{\partial Q_{1}}{\partial x} & =b \cdot W_{0} \\
\text { similarly, } \quad-\frac{\partial Q_{2}}{\partial x} & =b \cdot W_{0}
\end{array}\right\}
$$

Denoting salt concentrations by the same subscripts as densities, $c_{0}=0, c_{1}=\frac{p_{1}}{p_{0}}-1, c_{2}=\frac{p_{2}}{p_{0}}-1$, there must be no change in the mass of salt in any part of the estuary, and by definition, the mass of salt is c. (Mass of water), $=\Sigma c \cdot p \cdot Q \cdot \delta t=$ constant, therefore $\sum c \cdot p \cdot Q=0$. More preoisely, $\int_{t}^{t+T} c \cdot p \cdot Q . d t=$ constant. This is really a statement of Knudsen's hydrographic theorem.

Turning again to Fig. 8, we can draw up the aalt balance between sections 0 and $x$. Application of the above theorem gives

$$
c_{0} \cdot p_{0} \cdot Q_{0}+c_{1} \cdot p_{1} \cdot Q_{1}+c_{2} \cdot p_{2} \cdot Q_{2}=0 \text {. }
$$

Hence

$$
0+\left(\frac{p_{1}}{p_{0}}-1\right) \cdot p_{1} \cdot a_{1}+\left(\frac{p_{2}}{p_{0}}-1\right) \cdot p_{2} \cdot a_{2}=0 \text {. }
$$

On eliminating $Q_{2}$ by means of equation (5) and neglecting small quantities, we get 


$$
\left.\begin{array}{c}
Q_{1} \approx \frac{p_{2}-p_{0}}{p_{2}-p_{1}} \cdot Q_{R} \\
Q_{2} \approx-\frac{p_{1}-p_{0}}{p_{2}-p_{1}} \cdot Q_{R}
\end{array}\right\}
$$

whence

This pair of equations implies an infinite discharge at $x=I$ if $\left(\rho_{2}-\rho_{1}\right)$ is zero there, hence the restrictive clause after equation (4). Nevertheless it is instructive to use the density distributions (1) and (4) to calculate the variation of discharge with $x$. If we identify $P_{1}$ with the mean density $\bar{p}$, and take $p_{2}-p_{1} \approx \frac{1}{2} \cdot \delta_{p_{\text {Max }}} \cdot \sin \frac{\pi \cdot x}{L}=\delta_{p_{\text {Mx }}} \cdot \sin \frac{\pi x}{2 L} \cdot \cos \frac{\pi \cdot x}{2 L}$, substitution in equation (7) gives

$$
\left.\begin{array}{rl}
\frac{Q_{1}}{Q_{R}} & \approx 1+\frac{\Delta p}{\delta_{\rho M A x}} \cdot \tan \frac{\pi x}{2 . L} \\
-\frac{Q_{2}}{Q_{R}} & \approx \frac{\Delta p}{\delta_{\rho M A x}} \cdot \tan \frac{\pi \cdot x}{2 \cdot L}
\end{array}\right\}
$$

and

Curves of discharge as a function of $x$ are plotted in Figure 9 for the typical density ratio $\Delta p=15.8$ Max. They show the enormous increase in the rolume of water moring seawards, and the consequent counter drift, in a similar fashion to the curves deduced by Ketchum (1952) from a slightly different salinity distribution.

If the cross-sectional areas $A_{1}$ and $A_{2}$ (Fig. 8) were known, we could immediately calculate the mean velocities $\overline{\mathrm{U}}_{1}$ and $\overline{\mathrm{U}}_{2}$ as siretohed in Fig. 10, but unfortunately we know only the sum $A_{1}+A_{2}=A$ as a geometrical function of $x$. The arailable facta are $A_{1}=A_{R}$ and $A_{2}=0$ at $x=0$, and $A_{1} \approx A_{2}$ at $x=I$. The total depth $H$ is known but the height $h$ of the surface of zero net motion is determined by the bed roughness $z_{0}$ and the intensity of mixing, which depends on the tidal streams. Both $\overline{\mathrm{V}}_{1}$ and $\overline{\mathrm{V}}_{2}$ are of order $\bar{U}_{R}$.

If $b$ is known, the mean velocity of upwelling $\left(w_{0}\right)$ is readily determined from equation $(6)$. It is of order $\frac{H}{L} \cdot \frac{\Delta p}{\delta \rho_{\text {MAX }}} \cdot U_{R} \approx 0.001 U_{R}$, say $1 \mathrm{~mm} / \mathrm{sec}$., which exceeds the maximum rate of rise and fall of the water surface due to normal tidal motion, but is an order of magnitude below the root mean square value of the vertical fluctuations $w$. 


\section{TURBULENCE MEASUREMENTS}

Complete understanding of an estuary would need records of the variation of bed contours, water density, tide, shear stress, time-mean velocities $U, V$ and $W$, and the velocity fiuctuations $u, v$ and $w$, over shoals and channels throughout several tidal cycles. Civil engineers take many observations in nature, and in hydraulic models, to deal with the problems of dredging away sand bars and stabilising the flood and ebb channels along the approaches to a port. Oceanographers, meteorologists, and coastal engineers have amassed considerable data on water density, sediment concentration, tidal levels and discharges, and the influence of fresh water flow, wind action, and atmospheric pressure irregularities on long term averages.

Increasing attention is now being paid to the rapid fluctuations of velocity and other elements in tidal otreams, as a measure of fluid turbulence, but no investigator has yet measured simultaneously the velocity components in the three co-ordinate directions over the cross-section of a tidal channel. Several investigators have measured the longitudinal component $(U+u)$ and $a$ few the vertical fluctuation $w$ at fixed positions.

Prior to, and in the first years of the Second World War, German oceanographers were obtaining velocity records from a paddlewheel current meter anchored on the sea bed. In Norway, about 1947, experiments on bottom friction were conducted by the University of Bergen, using sensitive cup or bucket type current meters attached to a tripod resting on the sea bed.

In the United Kingdom, about 1948, a team from Iiverpool University made observations of $U$ and $u$ with Doodson pressure-operated current meters mounted in a stand or suspended from a boat in the Mersey, an example of a well-mixed estuary; here the R.M.S. value of $u$ averaged $0.05 \mathrm{U}$ to $0.10 \mathrm{U}$, without any clear trend in the rertical. Further observations, reported by Bowden and Fairbairn (1956) were carried out by the Iiverpool team in the Irish Sea near the coast of Anglesey, using electromagnetic flow meters fixed to a tripod on the sea bed, to record $u$ and w accurately, and U with less precision; the Reynolds stress - u.w. varied from 1 to $4 \mathrm{dyne} / \mathrm{cm}^{2}$ near the strength of flood or ebb. The large amount of numerical data on turbulent velocities resulting from later experiments with the electro-magnetic flow meters off Anglesey is being analysed by the DEUCE computer. Measurements at the same site by Bowden et al. (1959) of the time-mean velocity $U$ throughout the depth, at half-hourly intervals through the tidal cycle, using a Doodson meter suspended 
from the research vessel and cup-wheel meters on a tripod for the velocities just above the bed, indicated a systematic departure from the logarithmic profile due to phase differences between the velooities at each measuring point. However, this effect of tidal inertia was negligible close to the bed, say for the bottom $2 \mathrm{~m}$. In a total depth of $22 \mathrm{~m}$. , and here the logarithmic law

$$
\mathrm{U}=\frac{\mathbf{U}_{*}}{\mathrm{~K}} \cdot \ln \frac{z_{2}}{z_{0}}
$$

was obeyed, with von Karmán's constant $K=0.4$ and the roughness height $z_{0}=0.16 \mathrm{~cm}$. , corresponding to $k_{s} \approx 5 \mathrm{~cm}$. This equivalent sand roughness may be interpreted as due to ripples, for the bed consists of firm sand with small fragments of shell. The maximum value of bed shear tress $\tau_{0}=p$. U was about $8 \mathrm{dyne} / \mathrm{cm}^{2}$. The eddy viscosity raried in space and time; it was somewhat higher at mid-depth than nearer the surface or bed, and tended to maximum values when the tidal stream was at a maximum, numerical values of Nomentum being of the order of $270 \mathrm{~cm} .^{2} / \mathrm{sec}$. near strength of flood, and $130 \mathrm{~cm} . \mathrm{sec}$. near strength of ebb, when the depth-mean velocities were $\bar{U}=45 \mathrm{~cm} . / \mathrm{sec}$, and $\bar{U}=39 \mathrm{~cm} . / \mathrm{sec}$. respectively.

In the U.S.A., experiments by Iesser (1951) in which U was measured by four Ekman current meters suspended from a tripod in the lowest $2 \mathrm{~m}$. of water $45 \mathrm{~m}$. deep off the coast of California gave logarithmic velocity profiles with $z_{0}=0.1 \mathrm{~cm}$. Over sand which was

hydrodynamically rough, with maximum $\tau_{0}=5$ dyne/cm? and $z_{0}=0.02 \mathrm{~cm}$. over mud which behaved as a smooth boundary, with a maximum shear stress at the bed of only $0.2 \mathrm{dyme} / \mathrm{cm}^{2}$. In 1952, workers at Woods Hole Oceanographic Institution measured $U, u$, and $w$ (with less certainty) in the Kennebec estuary. Their turbulence meter was suspended from the research vessel, so observations near the water surface may have been distorted by its proximity. The R.M.S. values of $u$ and $w$ were of the same order, about $0.05, \mathrm{~J}$. High Reynolds stresses, of the order 10 to 30 dyne/cm., were associated with

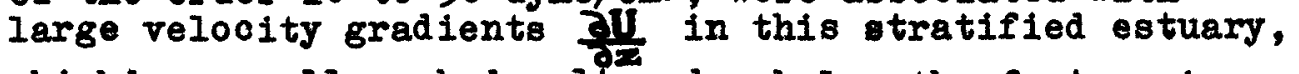
which has a well-marked salt wedge below the fast moving upper layer.

Turbulence measurements have been made for some jears in the rivers and estuaries of the Netherlands. While working with the Rijkswaterstaat in 1958, the author was able to measure the longitudinal velocity simultaneously with temperature and salinity, using an instrument designed by the Technical Physics Department (T.N.0.) for the Rijkswaterstaat, which is being des- 
cribed at the present Conference. Essentially this turbulence meter is a sensitive impeller, which responds rapidly to changes in water velocity, mounted on the otreamined body of an ott current meter weighing $100 \mathrm{~kg}$. It was suspended from a darit on the vessel "Christiaan Brunings" anchored at different positions in the Haringvilet. Observations were taken in one of two ways: either the instrument was steadily lowered to the bed, then winched to the surface, thus getting the vertical distribution over a short time interval, as in Fig. 11, or it was lowered in steps of 1 or $2 \mathrm{~m}$. and held for 2 ilinutes at each depth, giving a record of velocity against time at each depth, from which the time-mean velocity $U$ and the standard deviation $\sigma=\left(\bar{u}^{2}\right)^{1 / 2}$ could be estimated.

Results of the latter method of observation are plotted in Fig. 12 for flood and Fig. 13 for ebb streams. It will be noted that while the curvea of $U(z)$ are markedly different due to the net seaward flow near the surface, the curves of $\sigma(z)$ are alike, with peak values of $\sigma \approx 0.1 \bar{U}$ near the bed, similar to a river or other open channel with ateady flow. The vertical fluctuations were not measured, so the Reynolds otresses could not be determined. However, if $u$ and $w$ are of the ame order, we can estimate the mixing length $l$ from the formula

$$
l \cdot\left|\frac{\partial U}{\partial z}\right| \approx \sigma
$$

except where $U(z)$ passes through a maximum. There is not sufficient information to deduce the vertical distribution of $l$, but it seems to have a magnitude of order one tenth of the water depth. For purposes of calculation we will assume the mixing length distribution plotted in Fig. 14 is invariable throughout the tidal period. The curve in Fig. 14 has the equation

$$
\left.\begin{array}{ll}
l_{1}=k \cdot z \cdot \sqrt{\frac{H-z}{H-h}} & \text { for } H>z>h \\
l_{2}=k \cdot z & \text { for } h>z>0
\end{array}\right\}
$$

where von Kármán's constant $K \approx 0.4$ and the figure is plotted for the case $h=0.5 \widetilde{H}$.

\section{EQUATIONS OF MOTIOH}

z direction. Neglecting viscosity and the attractions of sun and moon, the vertical forces on a fluid element are due to hydrostatic pressure, gravity, and upwelling, the latter being very small. Application of Newton'a 
second law gives the vertical equation of motion:-

$$
\frac{d}{d t}(p \cdot W)+\frac{\partial p}{\partial z}+p \cdot g=0
$$

An approximate solution, satisfying the boundary conditions $p=p_{n}$ and $W=0$ at the free surface $(z=H)$, but neglecting the vertical density gradient and variations of W with time, is

$$
p=p_{A}+p \cdot g \cdot\left(H-z-\frac{W^{2}}{2 g}\right)
$$

Hence the longitudinal pressure gradient, assuming constant atmospheric pressure, is

$$
\frac{\partial p}{\partial x} \approx p \cdot g \cdot \frac{\partial H}{\partial x}+g \cdot(H-z) \cdot \frac{\partial p}{\partial x}
$$

$x$ direction. The longitudinal equation of motion, including friction but neglecting the tide-generating forces, is

$$
\frac{d}{d t}(p \cdot U)+\frac{\partial p}{\partial x}-\frac{\partial \tau}{\partial z}=0
$$

On expanding the first term, substituting equation (12) in the second term, and neglecting the rariation of density with time, equation (13) may be re-arranged thus

$$
\frac{\partial U}{\partial t}+U \cdot \frac{\partial U}{\partial x}+\frac{g \cdot(H-z)+U^{2}}{p} \cdot \frac{\partial \rho}{\partial x}-\frac{1}{p} \cdot \frac{\partial L}{\partial z}=-g \cdot \frac{\partial H}{\partial x}
$$

If the bed of the estuary is horizontal, the term on the right hand side represents the gravity component paraliel to the water surface. We will write $I=-\frac{\partial H}{\partial x}$ for the water surface slope, downwards from river to sea. This must equal the density-induced lope along the estuary, denoted by $D$, if all motion ceases; then equation (14) reduces to the simple form $\frac{H-z}{p} \cdot \frac{\partial p}{\partial x}=D$ assuming $\frac{1}{p} \cdot \frac{\partial \rho}{\partial x}=$ which can be integrated orer the vertical, flow conditions. Substituting $D(x)$ and $I(x, t)$ in equation (14) and changing the second term gives the working equation for this study:-

$$
\begin{aligned}
& \frac{1}{g} \cdot \frac{\partial U}{\partial t}+\frac{\partial}{\partial x}\left(\frac{U^{2}}{\partial g}\right)+2 D \cdot\left(1-\frac{z}{H}+\frac{U^{2}}{g H}\right)-\frac{1}{\beta g} \cdot \frac{\partial L}{\partial z}=I \\
& 10^{-5} \quad 10^{-6} \quad 10^{-6} \text { or } 10^{-7} \quad 10^{-5} \quad 10^{-5}
\end{aligned}
$$


ESTUARINE CURRENTS AND TIDAL STREAMS...

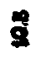

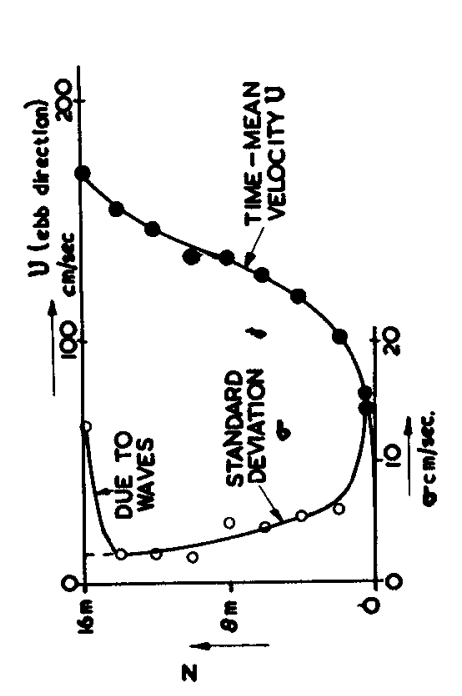

施

乐

$\frac{5}{5} \frac{8}{3}$

毫旁

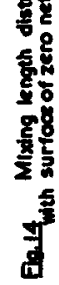

๖

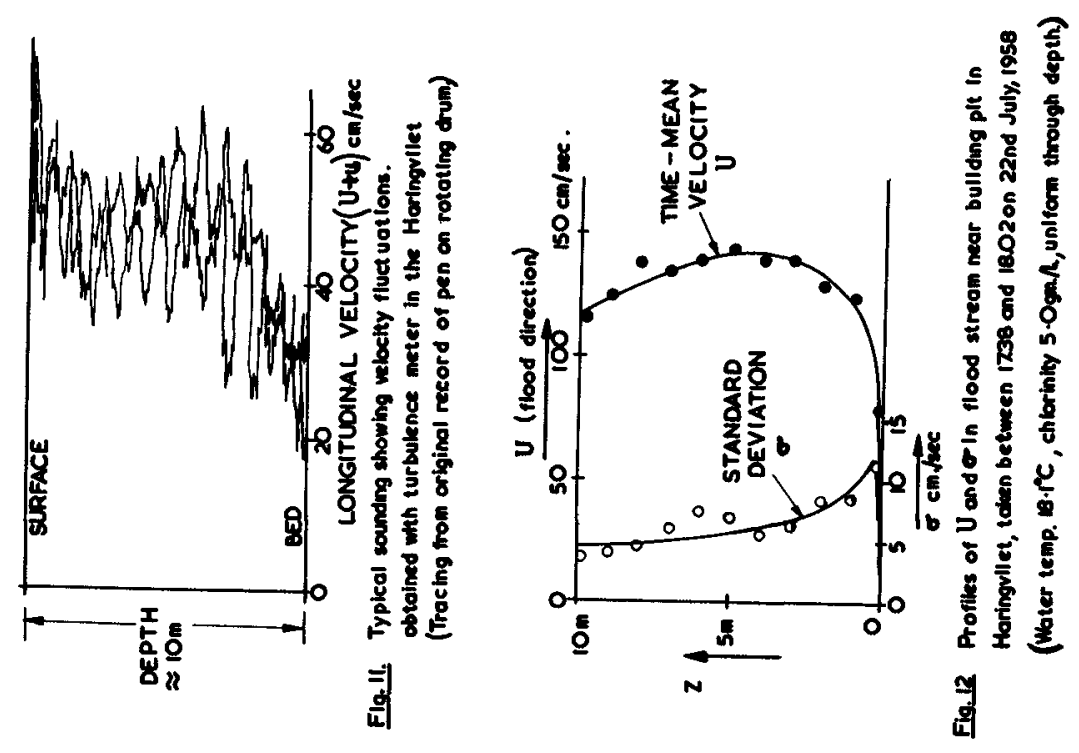


The order of magnitude of each term in a typical estuary is quoted. The surface slope $I \approx D+I$.cos $\omega t$ is balanced by components due to inertia, kinetic head, density gradient, and friction. The case where $D=0$ and the kinetic head is negligible has been solved. For laminar oscillations, Lamb (1932, p.622) gives the solution

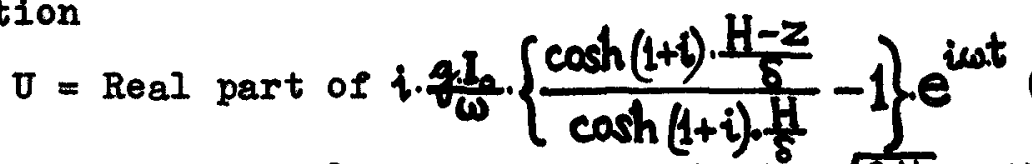

where the boundary layer thickness is $-\delta=\sqrt{\frac{2 y}{\omega}}$, the most noteworthy feature being a phase advance near the bed. Ionguet-Higgins (I953) has adapted this to find the mass transport under waves of finite amplitude, so correcting the frictionless theory of Stokes, and Abbott has applied this to a tidal estuary. For turbulent osoiliations in water of constant depth, theoretical and experimental investigations by Schonfeld (1948), McDowell (1955) and the author show that the velocity distribution is qualitatively like that predicted by Lamb.

In order to study the effects of a density gradient along the estuary, we seek a quasi-steady colution, $1 . e$. neglect the inertia term in (15) and assume the variation of kinetic head along the estuary is solely a density effect. As a first approximation take $\frac{\partial}{\partial x}\left(\frac{u^{2}}{2 g}\right)$ to have a constant value $F_{1}=\frac{\partial}{\partial x}\left(\frac{U_{1}^{2}}{2 y}\right)$ above the surface of
zero net motion, and another constant value $F_{2}=\frac{\partial}{\partial x}\left(\frac{U_{2}^{2}}{2 y}\right)$
below the surface of zero net motion, at any oross-sectioh $x$. We will moreover assume that the Froude number $\frac{U^{2}}{\text { Y.H }}$ is small, so the remaining part of equation (15) is

$$
F+2 D \cdot\left(1-\frac{z}{H}\right)-\frac{1}{p \cdot g} \cdot \frac{\partial F}{\partial z}=I
$$

This is an ordinary differential equation which can be integrated for the vertical distribution of shear stress. Using aubsoripts 1 and 2 for the layers, the boundary conditions to be satisfied are $\tau_{1}=0$ at $z=H, \tau_{1}=\tau_{2}$ at $z=h$, whence

and

$$
\left.\begin{array}{l}
\frac{\tau_{1}}{p \cdot g \cdot H}=1 \cdot\left(1-\frac{z}{H}\right)-D \cdot\left(1-\frac{z}{H}\right)^{2}-F_{1} \cdot\left(1-\frac{z}{H}\right) \\
\frac{\tau_{2}}{p \cdot g \cdot h}=1 \cdot\left(1-\frac{z}{H}\right)-D \cdot\left(1-\frac{z}{H}\right)^{2}-\frac{E \cdot(H-h)+F_{h} \cdot(h-z)}{H}
\end{array}\right\}
$$

At the bed, $\frac{\tau_{2}}{p \cdot g \cdot H}=I-D-F_{1}+\left(F_{1}-F_{2}\right) \cdot \frac{h}{H}=S$, say. Thus $\tau_{0}=p .8 . H . S$ and we see that the direction of the net bed shear stress is very sensitive to the values of $F_{1}, F_{2}$, and $h$, for $I \approx D$ at slack water. Fig. 10 shows that $F_{1}$ and $F_{2}$ may be of opposite sign in the upper reach. 
Writing $h=\alpha . H$, it will be shown that the fraction $\alpha$ is determined by $F_{1}, F_{2}$ and the roughness height $z_{0}$. In anticipation, we have plotted Fig. 15 for a crosssection where $\alpha=0.5, F_{1}=-3.6 \mathrm{D}, F_{2}=+4.0 \mathrm{D}$, and $I=D \cdot\left(10^{+} 10.00 s \omega t\right)$, at intervals of one lunar hour $\left(\omega t=30^{\circ}\right)$. As a second approximation, the discontinuity in the stress gradient $\frac{d \tau}{d z}$ at $z=h$ could be smoothed by making $F$ variable in each layer, with $F_{1}=F_{2}$ at $z=h$. Note the asymmetry in Fig. 15 despite the neglect of tidal inertia.

\section{VELOCITY DISTRIBUTION IN DENSITY CURRENT}

By definition, $\tau=p \cdot l^{2} \cdot\left|\frac{d U}{d z}\right| \cdot \frac{d U}{d z}$, except very close to the bed. Substitution in equation (18) with the mixing length distributions assumed in equation (9) gives the velocity gradient in each layer:-

$$
\frac{d U_{1}}{d z}= \pm
$$

$$
\frac{\sqrt{g \cdot h \cdot(1-\alpha) \cdot\left|R+D \cdot \frac{2}{H}\right|}}{K \cdot z}
$$

for $H>z>h$

and $\frac{d U_{2}}{d z}= \pm \frac{\sqrt{g H \cdot\left|S-2 \cdot P \cdot \frac{Z}{H}-D \cdot \frac{z^{2}}{H^{2}}\right|}}{K \cdot z}$ for $\left.h>z>z_{0}\right\}$ where $P=1 / 2 I-D-1 / 2 F_{2}, R=I-D-P_{1}$, and $S=I-D-$ $(I-\alpha) \cdot F_{7}-\alpha \cdot F_{2}$, as above. Re-writing (19) with the dimensiontess eleration $n=\frac{Z}{H}$ gives

$$
\left.\frac{K \cdot U_{1}}{\sqrt{g \cdot H}}= \pm \sqrt{1-\alpha} \cdot \int \frac{\sqrt{R+D . \eta}}{\eta} \cdot d \eta+\text { const. for } 1>\eta\right\rangle \alpha
$$

and

$$
\left.\frac{K \cdot U_{2}}{\sqrt{g \cdot H}}= \pm \int \frac{\sqrt{\left|S-2 \cdot P \cdot \eta-D \eta^{2}\right|}}{\eta} \cdot d \eta+\text { const. for } \alpha>\eta>z_{0}\right\}
$$

These are standard integrals but their solutions are too complicated for normal use. However, they may be simplified to yield the following approximations:-

$$
\begin{aligned}
& \frac{K \cdot U_{1}}{\sqrt{g \cdot H}} \approx \sqrt{(1-\alpha)|R|} \cdot\left\{ \pm \ln \frac{z}{h}+\frac{D}{|R|} \cdot \frac{z-h}{H}\right\}+\sqrt{|s|} \cdot\left\{ \pm \ln \frac{h}{z_{0}}-\frac{p}{|S|} \cdot \frac{h-z_{0}}{H}\right\} \\
& \text { and } \frac{k \cdot U_{2}}{\sqrt{g \cdot H}} \approx \sqrt{|S|} \cdot\left\{ \pm \ln \frac{z}{z_{0}}-\frac{P}{|S|} \cdot \frac{z-z_{0}}{H}\right\}
\end{aligned}
$$


COASTAL ENGINEERING
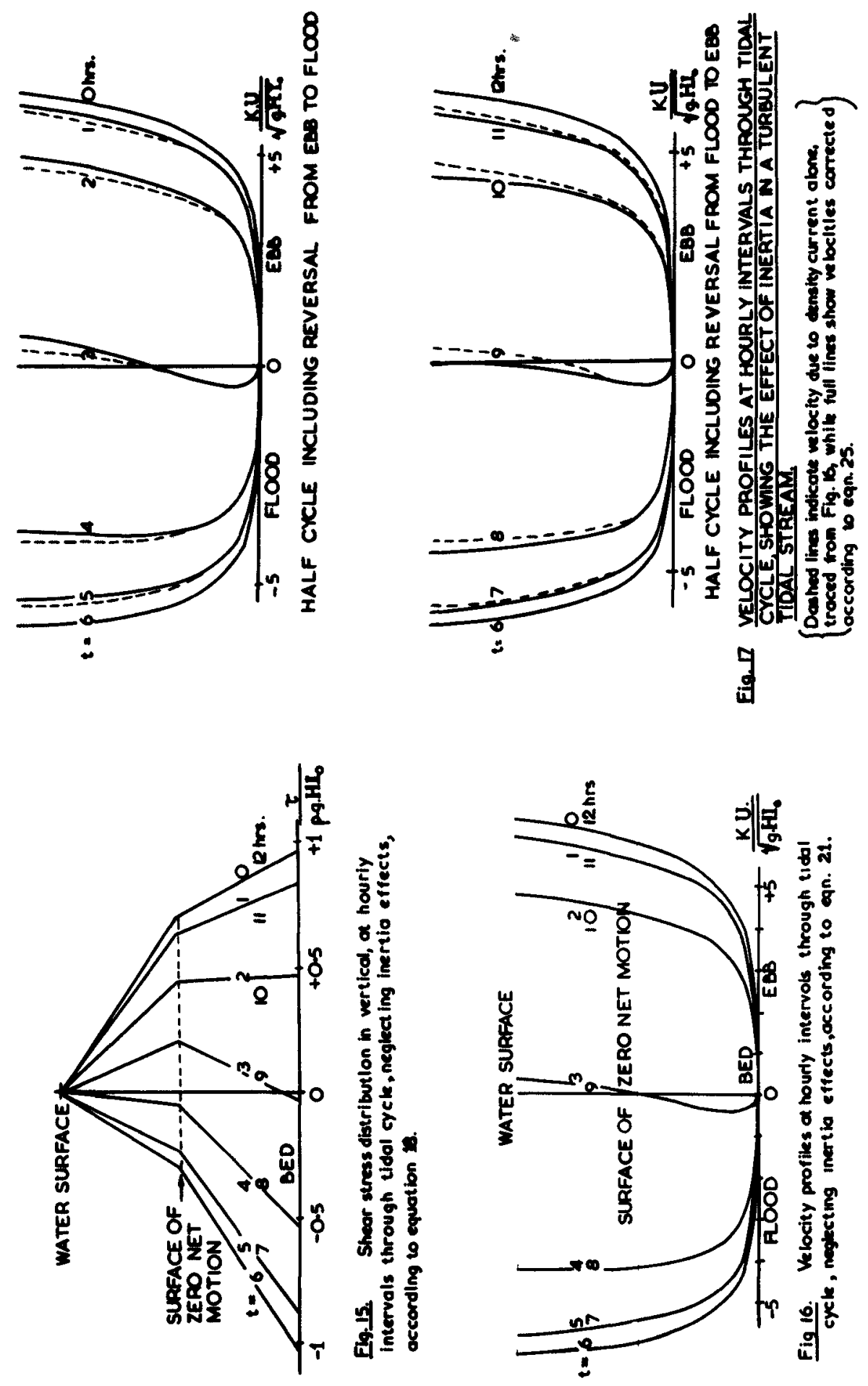
Take positive sign when $R, S>0$ (mainly ebb stream), and negative oign for $R, S<O$ (flood). The constants of integration were fixed by the boundary conditions of zero velocity near the bed $\left(U_{2}=0\right.$ at $\left.z=z_{0}\right)$ and continuity of the velocity profiles at the surface of zero net motion $\left(U_{1}=U_{2}\right.$ at $\left.z=h\right)$, where the velocities are identicaliy zero in the absenoe of tidal streams. This further condition produces the desired connection between $\alpha$ and $z_{0}$, for putting $v_{2}=0$ at $z=h=\alpha$. H gives

$$
\ln \left(\frac{\alpha \cdot H}{z_{0}}\right)=\frac{P}{S} \cdot\left(\alpha-\frac{z_{0}}{H}\right) \approx \frac{\frac{1}{2} \cdot D+\frac{1}{2} \cdot \Gamma_{2}}{(1-\alpha) \cdot F_{1}+\alpha \cdot F_{2}} \cdot \alpha
$$

writing $I=D$ and neglecting $z_{0}$ compared to $h$.

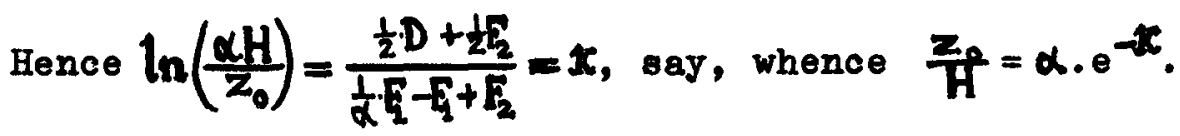

Since zo 18 omall, we can write as a first approximation

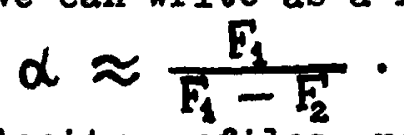

The resulting relocity profiles are plotted in Fig. 16, taking the previous figures for the "constants", implying that the roughness elements are such that

$\underline{z}_{0}=0.001$. Note the landward motion at slack water, due to the salinity current. The formulae (2I) reduce to Prandti's logarithmic velocity distribution

$U=\frac{(\mathrm{g} \cdot \mathrm{H} \cdot \mathrm{I})^{1 / 2}}{K} \cdot \ln \frac{\mathrm{z}}{\mathrm{z}_{0}}$ near strength of flood and $\mathrm{ebb}$, unless the tidal streams are unusually weak.

Other formulae have been devised by meteorologists to link departures from the logarithmic wind profile with temperature inversion (stable) or lapse (unstable) conditions. Atmospherle stability is characterised by the Richardson number, being the ratio of buoyancy force to inertia force. These semi-empirical formulae may be classified as linear, of type $\frac{d U}{d z}=m \cdot \frac{U_{*}}{k . z}$, or exponential, of type $\frac{d U}{d z}=\frac{U_{x}}{k \cdot z^{n}}$, where $m$ and $n$ are equal to unity in neutral conditions, and in general are functions of Richardson number. Thus Rosgby and Montgomery (1935) suggested $m=(I+\varepsilon . R i){ }^{1 / 2}$ where $\varepsilon=$ constant, and Deacon (1949) plotted his "profile index" $n$, showing a variation from about 0.8 for marked stability (Ri positive) to about 1.2 under unstable conditions (Ri negative). If $\mathrm{m}, \mathrm{n}$ are not functions of $\mathrm{z}$, integrate for the velocity profiles:- 
$v=\frac{m \cdot U_{*}}{k} \cdot \ln \left(\frac{z}{m \cdot z_{0}}\right)$

$$
\text { or } \begin{aligned}
\mathbf{U} & =\frac{u_{*}}{k} \cdot \frac{\left(\frac{z_{z}}{z_{0}}\right)^{1-n}-1}{1-n} \\
& \approx \frac{U_{x}}{k} \cdot\left\{\ln \frac{z}{z_{0}}+\frac{1-n}{2} \cdot\left(\ln \frac{z}{z_{0}}\right)^{2}\right\}
\end{aligned}
$$

The first formula merely implies a change in von Karman's "constant", so that the velocity profile remains logarithmic, contradicting many observations in density currents. The aecond formula implies a deformation of the simpie logarithmic profile, producing velocities concave to the ( $\ln z$ ) axis for unstable conditions (flood direction) and convex to the ( $\ln z$ ) axis for stable conditions (ebb direction for tidal current). The author's formula (21) causes a similar deformation of the logarithmic plot.

Recent work in the U.S.S.R., based on the idea of a layer of dynamic turbulence whose thickness $d$ is defined in terms of the local Richardson number as follows $\frac{1}{d}=\left(\frac{\partial R_{i}}{\partial z}\right)_{z \rightarrow 0}$, indicates that the eddy viscosity coefficient for non-neutral conditions in the atmosphere is simply $\mathbb{N}_{\text {Momentum }}=K \cdot U_{3 E} \cdot d$.Ri, and that a good first approximation for the velocity distribution is then

$$
\mathbf{U}=\frac{U_{z}}{k} \cdot\left\{\ln \frac{z_{1}}{z_{0}}+\beta \cdot \frac{z}{d}\right\} \text { for } z \gg z_{0}
$$

where the universal constants are $K \approx 0.4$ and $\beta \approx 0.6$, according to Monin and Obukhor $(1954)$. This is
precisely the author's equation $(21)$, if $\beta / \alpha$ is
identified with

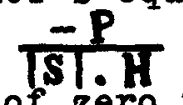
below the surface of zero net motion. It is interesting that $\frac{P}{S} \approx 0.5$ for large slopes, suggesting that $d$ is of the same order as the water depth $H$.

\section{EPFECTS OF THE NEGLECTED INERTIA TERM IN THE DYNAMIC EQUATION}

The neglected term $\frac{1}{g} \cdot \frac{\partial U}{\partial t}$ distorts the velocity profiles, especially near slack water, but it has no net effect if the tidal range is small compared to the water depth. Let us seek an inertial correction for the conditions of constant depth and simple harmonic motion. 
Omission of the first three terms in equation (15) leaves the equation of motion for uniform steady flow of a fluid with constant density:-

$$
-\frac{1}{\rho \cdot g} \cdot \frac{\partial \tau}{\partial z}=1
$$

This may be integrated twice, assuming that mixing length $l=k \cdot z \cdot\left(1-\frac{z}{H}\right)^{1 / 2}$, to give the logarithmic velocity profile $U(z)=\frac{\sqrt{4 H . I}}{K} \cdot \ln \frac{z}{20}$. Hence the simplest approximation to the velocity distribution in a tidal stream, where surface slope $I \approx I_{0} \cdot \cos \omega t$, is

$$
\begin{aligned}
U_{1}(z, t) & =\frac{\sqrt{g \cdot H \cdot I_{0}}}{k} \cdot \ln \frac{z_{0}}{z_{0}} \cdot \sqrt{ \pm \cos \omega t} \\
& \approx \sqrt{\frac{3 \pi}{8}} \cdot \frac{\sqrt{g \cdot H \cdot I_{0}}}{k} \cdot \ln \frac{z}{z_{0}} \cdot \cos \omega t
\end{aligned}
$$

The first term of the Fourier expansion has been taken, introducing the factor $\left(\frac{3 \pi}{8}\right)^{1 / 2}=1.08$. Note that it is impossible to have simple harmonic motion of both surface slope and tidal stream when the flow is turbulent. Also it is impossible to have S.H.M. of both tide and stream, even in a rectangular channel, so to this extent all solutions in this paper must be regarded as approximate.

Retention of the first term in equation (15) gives

$$
\frac{1}{g} \cdot \frac{\partial U}{\partial t}-\frac{1}{p \cdot g} \cdot \frac{\partial \tau}{\partial z}=I
$$

A trial solution is $U_{2}(z, t)=U_{0} \cdot \cos (\omega t-\phi)$, where $U_{0}$ and $\phi$ are functions of $z$. The velocity amplitude $U_{0}$ is well approximated by a logarithmic expression, so take $U_{0}=\frac{\left(\mathrm{g} \cdot \mathrm{H} \cdot \mathrm{I}_{0}\right)^{1 / 2}}{k} \cdot \ln \frac{\mathrm{z}}{\mathrm{z}_{0}}$. The phase lag $\phi$ will be assumed to be a linear function of $z$, although observations (e.g. Proudman, 1953, p.313, at Smith's Knoll) indicate that $\phi(z)$ is more nearly parabolic. If $\phi_{0}$ is the phase difference between velocities at surface and bed, we make the simple assumption that $\phi=\frac{z}{H}$. $\phi_{0}$, and we get the order of magnitude of $\phi_{0}$ from the value of $\phi_{d}=$ phase difference between mean velocity and bed shear stress or velocity gradient, as follows. Schonfeld (1948) applied the mixing length theory to compute the time difference $t_{d}$ between and $\tau_{0}$. His result, for a rough bed, is $t_{d}=\left(\frac{3.2 \mathrm{H}}{\mathrm{f}}\right)^{1 / 2}$, where $f=$ mean acceleration during reversal 
of tidal stream. If the motion is aimple harmonic, $f=\frac{\partial \widetilde{U}}{\partial t}=\omega \cdot \bar{U}_{0}$, whence the phase difference is

$$
\phi_{d}=\omega . t_{d}=\sqrt{\frac{32 \omega . H}{U_{0}}} \text { radians, or } \phi_{d}=360 \sqrt{\frac{3-2 H}{2 \pi \cdot U_{0}}} \text { degrees, }
$$

since $\omega=\frac{2 \pi}{T}$.

Just as Lamb's parameter $\frac{H}{\delta}$ governs the phase lags in laminar oscillations, it may be argued that a similar expression with $\delta$ defined by the eddy viscosity rather than the molecular viscosity will partly control the behaviour of turbulent fluid undereging simple harmonic motion. By analogy with $\frac{\mathrm{H}}{\delta}=\frac{\mathrm{H} \text { W }}{\sqrt{2 \nu}}$, we find the dimensionless ratio $\frac{H . \sqrt{\omega}}{\sqrt{\text { MOMENTUM }}} \propto \frac{\text { H. } \sqrt{\omega}}{\sqrt{H . U_{0}}} \propto \sqrt{\frac{H}{T_{.0}}}$ since eddy viscosity is proportional to $\mathrm{J}_{\pi}$, which is proportional to $\vec{U}_{0}$, in fact $\bar{U}_{z}=\frac{g^{1 / 2}}{c}$. $\bar{U}$ if $\mathrm{c}=$ Chezy coefficient for steady flow, and we assume that tidal flow depends on the maximum value $\vec{J}$ of the mean velocity in a vertical. Seeing this unique combination of water depth $H$, tidal period $T$, and mean velocity amplitude $0_{0}$, the author (1959) has defined the IAMB IUMBER as follows:-

$$
\mathcal{L}=\sqrt{\frac{44714 \text { 而 }}{1.0}}
$$

The factor 44714 is included to facilitate calculations on natural tidal oscillations, where the lunar semidiurnal period is 44714 sec., and to produce conveniently sized numbers. For example, the $\mathrm{M}_{2}$ tream in a channel of depth $10 \mathrm{~m}$. with maximum relocity $2 \mathrm{~m} . / \mathrm{sec}$. has $\mathcal{L}=2.24$. With this definition, schonfeldr formula for the phase difference over a rough bed reduces to the simple expressions:-

$$
\phi_{\mathrm{d}}=1.22 \mathcal{L} \text { degrees }
$$

Substitution in the abore example gives $\phi_{d}=2.7^{\circ}$. Hence the phase difference between surface and bed velooitios $\left(\phi_{0}\right)$ is of the order of 30 .

The effect of the inertia term $\frac{1}{g} \cdot \frac{\partial J}{\partial t}$ is to add a small velocity $\Delta U$ to the steady flow distribution. 


$$
\text { Thus } \begin{aligned}
\Delta U(z, t) & =U_{2}-U_{1} \\
& =U_{0} \cdot\left\{\cos (\omega t-\phi)-\left(\frac{3 \pi}{8}\right)^{1 / 2} \cdot \cos \omega t\right\} \\
& \approx 2 U_{0} \cdot \sin \frac{\phi}{2} \cdot \sin \left(\omega t-\frac{\phi}{2}\right), \text { neglecting }\left[\left(\frac{3 \pi}{8}\right)^{1 / 2}-1\right] \\
& \approx U_{0} \cdot \sin \phi \cdot \sin \omega t, \text { if } \phi \text { is small. }
\end{aligned}
$$

Hence we must apply the inertial correction (25) to quasi-steady velocity profiles:-

$$
\Delta \mathbf{v}=U_{0} \cdot \sin \phi \cdot \sin \omega t
$$

This correction has been applied to the velocities in (2I) to estimate the velocity distributions in the presence of both density and inertia effects, using the linear phase distribution $\phi=\frac{z}{H} \cdot \phi_{0}$ with $\phi_{0}=3^{\circ}$, and the previously assumed values of slope components and roughness ratio, giving Nikuradse $k_{\mathrm{s}} \approx 33 \mathrm{~cm}$. with

$H=10 \mathrm{~m}$, corresponding to a tidal channel with large sand ripples on the bed. Fig. 17 shows the resulting profiles. Although the velocity gradients near the bed, hence $\tau_{0}$, are increased after reversal of the tidal stream, they are correspondingly decreased before reversal, and the maximum value of $\tau_{0}$ appears to be the same as that obtained when inertia is neglected, with this "slowly varled" flow; only at very short tidal periods, $a s$ in hydraulic models, is there a measurable increase in the maximum bed shear stress. However, the phase difference between velocity and water surface slope is by no means negligible, so linear superposition of the quasi-steady and inertial solutions of the dynamic equation cannot produce very reliable results. But here we are interested in the general behaviour of estuarine water, so further refinement in the correction $\Delta U$ will not be attempted.

Before leaving this subject, it should be emphasized that the phase differences inside the fluid, al though small, may be important for the proper operation of tidal models with movable bed material, since grains set in motion relatively early in the tidal cycle may continue moving with the main stream even when the bed shear stress has fallen below the value required to initiate movement. For reproduction of inertia effects the Iamb number should be the same in model and prototype (unless the friction coefficient differs), calling for models without vertical exaggeration if the Froude scale law is followed. 


\section{EFFECTS OF FINITE TIDAI RANGE}

If the tidal range is of the same order as the water depth, as occurs in many shallow estuaries, then the second term in equation (15) cannot be wholly attributed to the salinity circulation and it may change sign along an estuary due to changes in bottom topography (contractions and expansions) as well as the water surface profile varying in time and space. We must therefore apply a further correction to the velocity distributions at any cross-section.

Longuet-Higgins (1953) showed that at elevation $\delta=\left(\frac{2 y}{\omega}\right)^{\not / 2}$ above a smooth bed, there was a net velocity in the direction of ware propagation, equal to

$$
\frac{5}{4} \cdot \frac{\omega^{2} \cdot a^{2}}{c_{0} \cdot \sinh ^{2} \frac{\omega H}{c_{0}}} \approx \frac{5}{4} \cdot\left(\frac{a}{H}\right)^{2} \cdot c_{0}
$$

with "long" progressive waves, where $a=$ amplitude, and $c_{0}=$ celerity of wave. Experiments indicate that the net forward velocity over a rough bed is lower than this figure. The mass transport under a standing wave is smaller and distributed differently in the vertical. The case of a tidal estuary, which may be treated as a channel closed at one end and open to the sea at the other end, involves the combination of an incident wave whose amplitude decreases exponentially in the direction of propagation (from open to closed end), and a reflected wave whose amplitude decreases exponentially from the closed end to the sea. A general solution is very difficult, but the direction of net movement in a tidal estuary may be inferred from available data on the variation of tidal elevations and mean velocities along the estuary.

Abbott (1960) has suggested the criterion $\frac{d}{d x}\left(\bar{U}_{0} \cdot e^{\theta}\right)$ $=0$ for finding the positions along a tidal estuary where sediment collects, so explaining the Mud Reaches in the Thames estuary. Here $\overline{\mathrm{U}}_{0}=\operatorname{maximum}$ velocity of tidal stream, and $\theta=$ phase difference between tide and stream.

\section{REFERENCES}

Abbott, M.R. : Boundary Iayer Effects in Estuaries, Journal of Marine Research, 1960.

Agnew, R. : Tidal otreams in natural and artificial channels, Ph.D. thesis, Queen's University, Belfast, 1959. 
Bowden, K.F. and Fairbairn, I.A. : Measurements of turbulent fluctuations and Reynolds stresses in a tidal current, pp. 422-438, Vol.A.237, Proc. Roy. Soc., 1956.

Bowden, K.F., Fairbairn, L.A. and Hughes, P. : The distribution of shearing stresses in a tidal current, pp. $288-305$, Vol. 2, Geophysical Journal, 1959.

Deacon, E.L. : Vertical diffusion in the lowest layers of the atmosphere, pp. 89 - 103, Vol. 75, Q.J. Roy. Met. Soc., 1949.

Inglis, C.C. and Allen, F.H. : The regimen of the Thames estuary as affected by currents, salinities, and river flow, pp. $827-868$, Vol. 7 , Proc. I.C.E., 1957.

Ketchum, B.H. : Circulation in estuaries, pp. $65-76$, Proc. 3rd Conf. Coast. Eng., 1952.

Lamb, H. : Hydrodynamics, C.U.P., 6th ed., 1932.

Lesser, R.M. : Some observations of the velocity profile near the sea floor, pp. 207 - 211, Vol. 32, Tr. A.G.U., 1951.

Longuet-Higgins, M.S. : Mass transport in water waves, pp. $535-581$, VoI. A. 245, Phil. Trans. Roy. Soc., 1953.

McDowell, D.M. The vertical distribution of water velocities in tidal streams and in models of tidal regions, Paper A.1, Proc. I.A.H.R., The Hague, 1955 .

Monin, A.S. and Obukhov, A.M. : Basic regularity in turbulent mixing in the surface layer of the atmosphere, U.S.S.R. Acad. Sci., Works of the Geophysical Institute, No. 24 (151) in Russian, 1954.

O'Brien, M.P. Salinity currents in estuaries, pp. 520 522, Vol. 33, Tr.A.G.U., 1952.

Otter, J.R.H. and Day, A.S. : Tidal flow computations, pp. 177 - 182, Vol. 209, "The Engineer", 29 th Januar:, 1960.

Pillsbury, G.B. : Tidal hydrualics, U.S. Army Corps of Engineers, revised ed., 1956.

Proudman, J. : Dynamical oceanography, Methuen, 1953. 
Rossby, C.G. and Montgomery, R.B. : The layer of friotional Influence in wind and ocean currents, Vol. 3, Papers in Physical Oceanography and Meteorology, Cambridge, Mass., 1935.

Schunfeld, J.C. : Resistance and inertia of the flow of liquids in a tube or open canal, pp. 169 197, Vol. A.1, Applied Sci. Res., 1948.

Takano, K. : On the salinity and the velocity distributions off the mouth of a river, pp. 60 67, Vo1. 10, J. Oceanogr. Soc., Japan, 1954.

Taylor, G.I. : Internal waves and turbulence in a fluid of variable density, pp. 35 - 42, Vol. 76 , Rapports et Process-Verbaux des Réunions du Conseil Permanent International pour l'Exploration de la Mer, 1931.

\section{NOTATION}

A area of cross-section

a amplitude of long wave

$b$ breadth of surface of zero net motion

C Chezy coefficient of friction

c concentration of salt by weight

co celerity of long wave, $=(\mathrm{g} \cdot \mathrm{H})^{)_{2}}$ in the absence of friction

D slope component due to longitudinal density gradient, $=\frac{H}{2 p} \cdot \frac{\partial p}{\partial x}$

d thickness of turbulent boundary layer in atmosphere

e base of natural logarithms, $=2.718$

F slope component due to longitudinal velocity

$$
\text { gradient, }=\frac{\partial}{\partial x}\left(\frac{\mathrm{J}^{2}}{2 g}\right)
$$

1 acceleration during reversal of tidal stream

8 acceleration due to gravity water depth

elevation above bed of surface of zero net motion surface slope, taken positive downwards to sea imaginary number, defined by $1^{2}=-1$ number defined in text

sand grain diameter in Nikuradse's experiments Lamb number length of estuary

mixing leng th

$n$ numbers defined in text

$\mathrm{N}$ eddy coefficlent

$P$ slope component defined in text

p pressure

Q discharge $=$ volume per unit time 
Ri Richardson number

$R, S$ slope components defined in text

T tidal period

$t$ time

J,u time-mean and fluctuating velocities, respectively, in $x$-direction

$\mathrm{V}, \mathrm{v}$ time-mean and fluctuating velocities, respectively, in $y$-direction

$W, w$ time-mean and fluctuating velocities, respectively, in $z$-direction

$X$ fluid displacement in $x$-direction

$x$ longitudinal distance, positive from river to sea

$y$ transverse distance

$z$ vertical distance, positive upwards

zo roughness height

$\alpha$ dimensionless elevation $\frac{h}{H}$ of surface of zero net motion

$\beta$ Obukhor's constant $\approx 0.6$

$\gamma$ ratio of eddy coefficients of riscosity and diffusion

$f$ thickness of laminar boundary layer in osoiliating

$$
\text { flow, }=\left(\frac{2 \psi}{\omega}\right)^{1 / 2}
$$

$\varepsilon$ "constant" introduced by Rossby and Kontgomery

$\eta$ dimensionless elevation $\frac{z}{H}$ above bed

$\theta$ phase difference between tide and tidal stream

$k$ von Karman's constant $\approx 0.4$

$\nu$ kinematic viscosity of fluid

$p$ density of fluid

o standard deviation of turbulent velocity $u$

c shear stress at elevation $z$

$\tau_{0}$ shear stress at bed

$\phi$ phase lag of velocity

$\omega$ angular velocity of tidal $s$ tream $=\frac{2 \pi}{1}$ 